\title{
Agrupación difusa como herramienta para reducir la subjetividad en el diagnóstico de aisladores poliméricos
} Fuzzy clustering as a tool for reducing subjectivity
in the diagnosis of polymer insulators

João Pedro da Costa Souza ${ }^{1}$, Edson Guedes da Costa², Luiz Augusto Medeiros Martins Nobrega ${ }^{3}$, Bruno Albuquerque Dias ${ }^{4}$, Antonio Francisco Leite Neto ${ }^{5}$

da Costa Souza, J.P; Guedes da Costa, E; Medeiros Martins Nobrega, L.A; Albuquerque Dias, B; Leite Neto, A.F. Agrupación difusa como herramienta para reducir la subjetividad en el diagnóstico de aisladores poliméricos. Tecnología en Marcha. Vol. 34, especial. ALTAE. Diciembre 2021. Pág 193-204.

doi) https://doi.org/10.18845/tm.v34i7.6041

1 Universidade Federal de Campina Grande - UFCG. Brasil. Correo electrónico: joao.souza@ee.ufcg.edu.br

(iD) https://orcid.org/0000-0002-3490-6762

2 Universidade Federal de Campina Grande - UFCG. Brasil. Correo electrónico: edson@dee.ufcg.edu.br

(iD) https://orcid.org/0000-0003-2998-2039

3 Universidade Federal de Campina Grande - UFCG. Brasil. Correo electrónico: luiz.nobrega@dee.ufcg.edu.br

(iD https://orcid.org/0000-0003-2634-8526

4 Universidade Federal de Campina Grande - UFCG. Brasil. Correo electrónico: bruno.dias@ee.ufcg.edu.br

(iD https://orcid.org/0000-0001-9521-6864

5 Universidade Federal de Campina Grande - UFCG. Brasil. Correo electrónico: antonio.leite@ee.ufcg.edu.br 


\title{
Palavras-chave
}

Diagnóstico de isoladores; isoladores poliméricos; agrupamento difuso; redução da subjetividade; estado operacional.

\section{Resumo}

O diagnóstico das condições operacionais de isoladores poliméricos é um tema pertinente de estudo e pode apresentar elevada carga de subjetividade. Assim, o presente trabalho tem como objetivo propor uma metodologia, baseada em agrupamento difuso (fuzzy clustering), para reduzir a subjetividade no diagnóstico de isoladores poliméricos. Foram ensaiados 60 isoladores poliméricos de tensão nominal de 138 kV com diferentes níveis de degradação. Os isoladores foram classificados inicialmente a partir de inspeção visual em três níveis de degradação: baixo, intermediário e crítico. Em seguida, os isoladores foram submetidos às seguintes técnicas de monitoramento: detecção de radiação ultravioleta, termografia infravermelha e detecção de ruído ultrassônico. Os dados oriundos das inspeções foram extraídos e processados. Para cada método de inspeção, as amostras foram agrupadas em três conjuntos a partir de agrupamento difuso. Os conjuntos foram então associados a cada nível de degradação a partir da análise minuciosa dos dados. Um diagnóstico foi estabelecido considerando os agrupamentos relativos às três técnicas utilizadas e os resultados foram comparados ao diagnóstico oriundo da inspeção visual, sendo o estado mais crítico entre os dois considerados. Constatou-se que 5 dos isoladores apresentaram nível de degradação mais elevado do que o estabelecido a partir de inspeção visual, de modo que a metodologia utilizada asseguraria a retirada ou supervisão mais conservadora dos isoladores. A metodologia proposta reduz a subjetividade do diagnóstico de isoladores poliméricos, inserindo um fator não supervisionado na classificação do seu estado operacional.

\section{Keywords}

Diagnosis of insulators; polymer insulators; fuzzy clustering; reduction of subjectivity; operational state.

\begin{abstract}
The diagnosis of polymer insulators is a relevant topic of study and may present a high subjectivity. Thus, the present work aims to propose a methodology based on fuzzy clustering to reduce subjectivity in the diagnosis of polymer insulators. 60 polymer insulators with different degradation levels and nominal voltage of $138 \mathrm{kV}$ were tested. The insulators were classified through visual inspection in three degradation levels: low, intermediate and critical. Then, the insulators were submitted to the following monitoring techniques: ultraviolet radiation detection, infrared thermography and ultrasound noise detection. Data from inspections were extracted and processed. For each monitoring method besides visual inspection, samples were grouped in three clusters. The clusters were associated to the degradation levels through detailed data analysis. A diagnosis was stablished considering the clusters from the three monitoring methods and results were compared to the diagnosis considering the visual inspection, and the most critical condition among them was considered. Five of the insulators presented a higher degradation level than the one stablished through visual inspection, so the methodology used would ensure the removal or supervision of the equipment. The proposed methodology reduces subjectivity in the diagnosis of polymer insulators while adding an unsupervised factor in the classification of its operational state.
\end{abstract}




\section{Introdução}

Isoladores poliméricos têm substituído gradativamente isoladores cerâmicos em linhas de transmissão desde o decênio de 1980 em razão de diversas vantagens, tais como menor peso, custo e maior resistência à contaminação [1],[2]. Apesar das vantagens, existe carência de informações relativas ao processo de formação de falhas, como o envelhecimento dos materiais poliméricos e influência da exposição contínua à poluição, o que fomenta a necessidade do uso de técnicas inovadoras para garantir a disponibilidade e confiabilidade das linhas [3-5].

Algumas das principais técnicas utilizadas para o monitoramento de isoladores poliméricos são a detecção de radiação ultravioleta, a termografia infravermelha, a detecção de ruído ultrassônico e a inspeção visual. Ainda que confiáveis, tais técnicas apresentam limitações. A inspeção por detecção de radiação UV carece de padronização e da determinação de parâmetros significativos para identificação do estado de degradação do isolador [6],[7]. Inspeções por termografia infravermelha e detecção de ruído ultrassônico, por sua vez, apresentam grande influência do ambiente, ainda não completamente mensurada [8]. Por fim, a técnica de inspeção mais comum - a inspeção visual - depende da experiência e senso crítico do avaliador [9]. Ainda que algumas dessas limitações possam ser mitigadas pela utilização das técnicas em conjunto, a falta de padronização e de critérios objetivos na maior parte dos métodos de inspeção insere um grau de subjetividade no diagnóstico de isoladores poliméricos.

Nesse contexto, a associação de técnicas de monitoramento a modelos de aprendizado de máquina para determinação do estado operacional de isoladores poliméricos surge como uma alternativa para reduzir a subjetividade em seu diagnóstico [10]. O aprendizado de máquina pode ser dividido em aprendizado supervisionado e não supervisionado. No aprendizado nãosupervisionado, o objetivo é a determinação de estruturas no banco de dados: a identificação de possíveis padrões, sem a necessidade de rótulos prévios explícitos. De forma resumida, busca-se a identificação de uma possível relação entre dados disponíveis, com associação de tendências. No aprendizado supervisionado, exemplos de entradas de dados e suas respectivas saídas são fornecidos em uma etapa de treinamento de modelos, de modo que se torna possível mapear o comportamento das saídas em função das entradas a partir de uma regra geral [11].

Diversos trabalhos se propuseram ao diagnóstico de isoladores poliméricos auxiliado por aprendizado supervisionado [12-16]. Entretanto, nesses trabalhos, a subjetividade permeia o diagnóstico desde a etapa de treinamento, realizada a partir de amostras classificadas por inspeção visual. Embora seja impossível eliminar completamente a subjetividade do diagnóstico, métodos que reduzam essa subjetividade são desejáveis. Uma abordagem possível é a utilização de metodologias baseadas em técnicas de aprendizado de máquina não supervisionado [17], como o agrupamento difuso.

Agrupamento (ou clustering, no inglês) é uma tarefa de aprendizado de máquina não supervisionado que tem como objetivo agrupar objetos em clusters, de forma que objetos similares estejam contidos em um mesmo cluster e objetos diferentes estejam em clusters diferentes. O agrupamento é uma das tarefas mais utilizadas em análise exploratória de dados e pode ser realizado a partir de diferentes algoritmos, sendo um dos mais comuns o agrupamento difuso (ou fuzzy clustering, no inglês).

O agrupamento difuso cria classificações intermediárias (a partir de coeficientes de partição, que indicam o grau de pertencimento a um cluster e cuja soma deve ser igual a 1), diferentemente de outras formas de agrupamento, que atribuem um elemento unicamente a um determinado cluster. Assim, o agrupamento difuso também é algumas vezes denominado de agrupamento suave (soft clustering, no inglês) [18]. O agrupamento difuso é bastante útil quando fronteiras entre clusters são ambíguas e não estão bem definidas. Além disso, a afiliação a múltiplos 
clusters pode auxiliar na descoberta de relações mais sofisticadas entre clusters para um dado conjunto de dados [19]. Esse tipo de agrupamento pode ser de grande utilidade no diagnóstico de isoladores, dado que progressões em seu estado operacional ocorrem de forma contínua.

Assim, o presente trabalho tem como objetivo propor uma metodologia baseada em agrupamento difuso de forma a reduzir a subjetividade no diagnóstico de isoladores poliméricos, levando em consideração informações oriundas da inspeção visual. Nesta seção, o tema central de estudo foi introduzido e discutido, de forma a apresentar as motivações do presente trabalho, destacando-se o seu objetivo. Na seção 2, Metodologia, o material utilizado e os métodos desenvolvidos para alcançar o objetivo proposto são apresentados. Na seção 3 , os resultados obtidos são expostos e discutidos. Por fim, na seção 4, as conclusões alcançadas são apresentadas; trabalhos futuros são sugeridos. Este trabalho se encerra com a prestação dos devidos agradecimentos e a apresentação das referências utilizadas.

\section{Metodologia}

\section{Materiais}

Os isoladores utilizados nos ensaios em laboratório podem ser observados na figura 1. As amostras correspondem a 60 isoladores poliméricos de $138 \mathrm{kV}$, retirados de operação da linha que interliga os municípios de Campina Grande - PB e Santa Cruz - RN, Brasil.

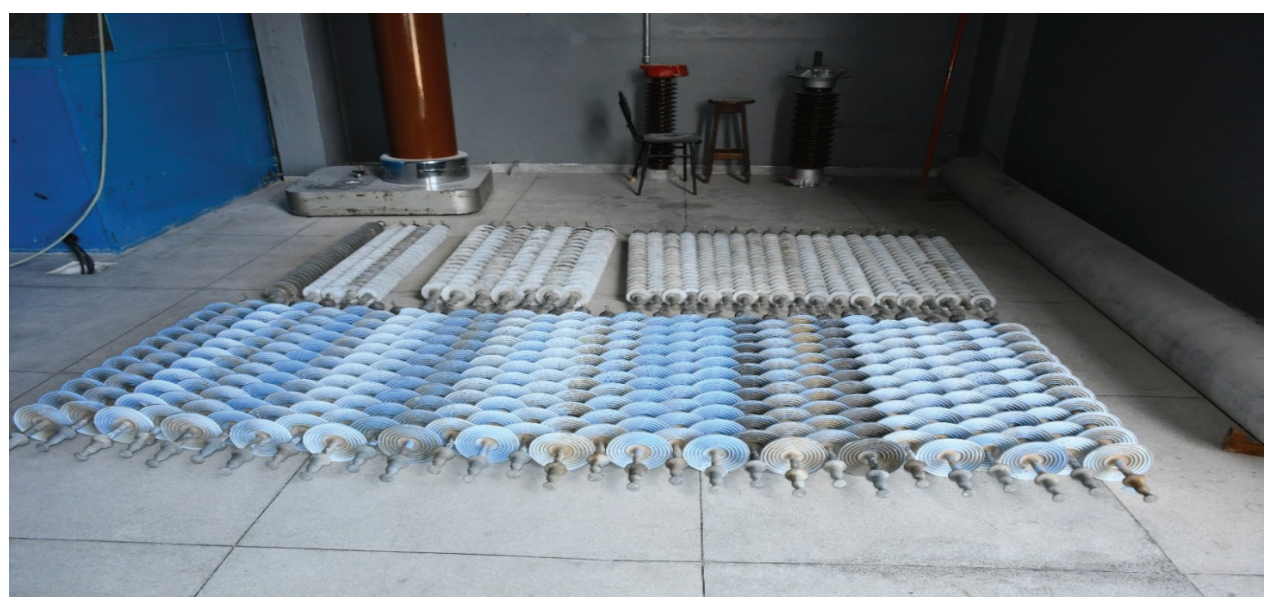

Figura 1. Fotografia dos isoladores poliméricos utilizados neste trabalho.

Os isoladores analisados possuem tipos distintos de revestimento polimérico, de EPDM e silicone, com terminais metálicos similares (tipo concha no terminal terra e bola no terminal fase), fabricados em aço galvanizado; o núcleo é composto por fibra de vidro reforçada com resina epóxi.

\section{Métodos}

Os isoladores poliméricos sob estudo foram inspecionados visualmente e ensaios em laboratório foram realizados para extração de dados a partir das seguintes técnicas de monitoramento: inspeção por detecção ultravioleta; termografia infravermelha e detecção de ruído ultrassônico. Os dados foram processados e os isoladores foram diagnosticados de acordo com a metodologia baseada em agrupamento difuso proposta neste trabalho. Os métodos utilizados são elucidados a seguir. 


\section{Inspeção visual}

Os isoladores foram inicialmente classificados a partir de inspeção visual, de acordo com diretrizes observadas na bibliografia especializada. A partir dos métodos propostos em [9] e [20], sugeriu-se o sistema de classificação apresentado na tabela 1.

Tabela 1. Classes de isoladores poliméricos sugeridas neste trabalho.

\begin{tabular}{|c|c|c|}
\hline \multicolumn{3}{|c|}{ Classificação sugerida } \\
\hline Classes & Nível de degradação & Descrição \\
\hline A & Baixo & Isoladores com baixo ou nenhum risco \\
\hline B & Intermediário & Isoladores com risco moderado \\
\hline C & Crítico & Isoladores com risco crítico \\
\hline
\end{tabular}

Ensaios em laboratório e extração de dados

Ensaios em laboratório foram conduzidos para extração de informações relativas à termografia infravermelha, radiação ultravioleta e ruído ultrassônico. A corrente de fuga também foi medida para fins de controle. Uma fotografia do arranjo utilizado é apresentada na figura 2.

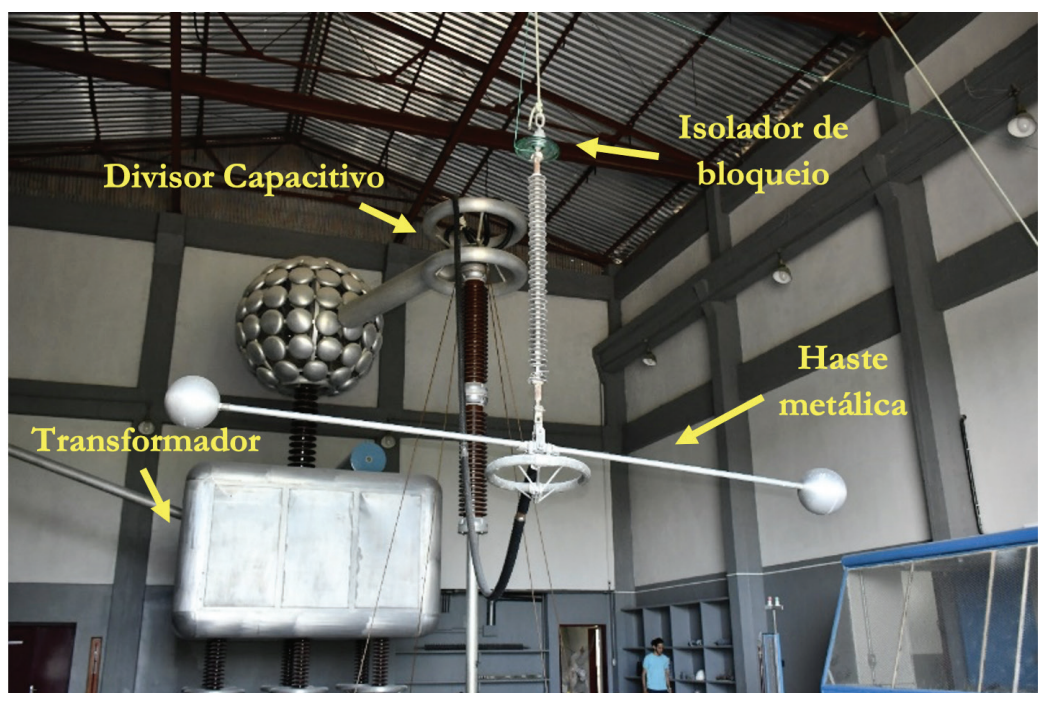

Figura 2. Fotografia do arranjo em laboratório.

Os métodos de inspeção por detecção de radiação ultravioleta, termografia infravermelha e detecção de ruído ultrassônico foram empregados a partir de uma plataforma localizada a 8 $\mathrm{m}$ dos objetos de teste. Os equipamentos utilizados para cada um dos métodos supracitados foram uma câmera de detecção UV modelo DayCor II@ da Ofil Systems, um termovisor Systems P65, da FLIR e um detector de ruído ultrassônico Ultraprobe 2000 da UE Systems.

A partir do método de inspeção por termografia infravermelha, pelo menos três imagens térmicas foram registradas para cada isolador. Um vetor de temperaturas foi extraído do corpo dos isoladores. O aumento da temperatura dos isoladores, a variação de temperatura ao longo dos isoladores ( $1^{a}$ derivada do vetor de temperaturas) e a variação entre variações de temperaturas ( $2^{a}$ derivada do vetor de temperaturas) foram extraídos. Ademais, operações de estatística descritiva foram realizadas para extração de atributos como medidas de tendência central e de dispersão, iniciativa similar à proposta em [13]. Ao todo, 33 atributos de temperatura foram extraídos. 
A partir do método de inspeção por detecção de radiação ultravioleta, dois vídeos de 1 (um) minuto de duração foram registrados para cada isolador polimérico. A extração dos atributos oriundos das inspeções por detecção de radiação ultravioleta foi realizada a partir da metodologia proposta em [6], sendo extraídos os seguintes atributos: persistência das descargas ao longo do vídeo, a área das descargas (número de pixels associados à manifestação UV), o perímetro das descargas (número de pixels correspondente às bordas da manifestação UV) e o fator de forma (razão entre área e perímetro de descargas).

A partir do método de inspeção por detecção de ruído ultrassônico, três sinais de áudio de 30 segundos de duração foram registrados. A extração dos atributos oriundos das inspeções por detecção de ruído ultrassônico foi realizada para cada uma das três amostras de áudio registradas. Os atributos obtidos foram comparados entre si e o atributo mais distinto foi excluído. A média entre os dois atributos mais similares foi utilizada como o atributo final. Essa estratégia foi utilizada de modo a excluir amostras possivelmente afetadas por ruídos externos ou falhas na medição. O processamento dos sinais de ruído ultrassônico abrangeu operações de estatística descritiva, extração de harmônicas, vetores de Energia dos Centróides de Subbandas Espectrais (VECSE) e a transformada Wavelet dos sinais, iniciativa similar às propostas em [16], [21] e [23]. Para todos os métodos de inspeção, os outliers foram retirados.

\section{Metodologia proposta baseada em agrupamento difuso}

O processo de agrupamento difuso foi realizado isoladamente para cada método de inspeção utilizado, com exceção da inspeção visual. A associação dos clusters ao estado operacional dos isoladores foi realizada a partir da análise dos seus centroides. A seguir, tendo em vista que a seleção de atributos pode ajudar na melhoria de algoritmos de aprendizado nãosupervisionado [24], uma seleção prévia de atributos foi realizada com base na correlação dos atributos entre si (redundância). Os atributos que possuíam correlação elevada com outros atributos foram considerados redundantes, sendo retirados a partir de um processo similar ao realizado em [25]: os atributos foram ordenados a partir da correlação média com outros atributos e os atributos mais redundantes foram retirados. Tendo em vista o menor número de atributos oriundos das inspeções por detecção de radiação ultravioleta, a seleção prévia de atributos não foi realizada para os dados desse tipo de inspeção.

Ao fim da etapa não supervisionada, coeficientes de partição foram considerados notas. As notas relativas a cada método de inspeção foram somadas para cada nível de degradação, em um sistema similar a uma votação. Os isoladores foram classificados de acordo com o nível de degradação associado à maior nota.

Por fim, a classificação oriunda da etapa não supervisionada foi comparada à classificação inicial por inspeção visual e o estado mais crítico dentre as duas classificações foi considerado. Por exemplo, um isolador que tenha sido classificado como A na etapa não supervisionada e como B na classificação por inspeção visual teria B como rótulo final.

\section{Resultados}

\section{Inspeção visual}

Os 60 isoladores poliméricos foram classificados visualmente nas classes A, B e C, descritas na tabela 1. Exemplos de isoladores classificados em cada classe podem ser observados na figura 3, com destaque para alguns dos defeitos mais evidentes observados. 


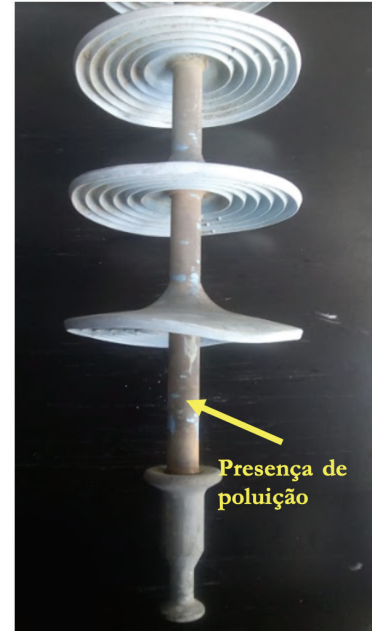

(a)

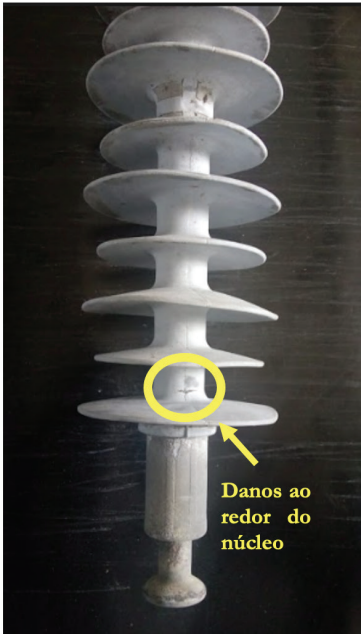

(b)

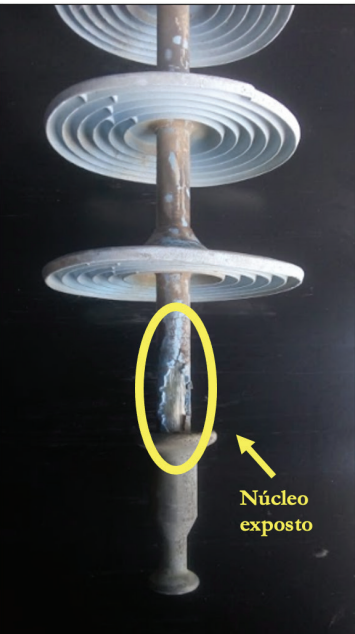

(c)

Figura 3. Fotografias de isoladores classificados via inspeção visual como pertencentes à Classe $A, B$ e $C$.

Na figura 3 (a) tem-se um isolador atribuído visualmente à classe A. Nota-se a presença de uma leve camada de poluição; no isolador apresentado na figura 3 (b), é possível observar danos crescentes no revestimento polimérico, porém sem exposição do núcleo de fibra de vidro, o que motivou a sua associação à classe B. Por fim, na figura 3 (c), um isolador atribuído visualmente à classe $\mathrm{C}$, que representa a condição operacional mais crítica dos isoladores, em que a exposição do núcleo de fibra de vidro é perceptível e os isoladores devem, assim, ser retirados de operação urgentemente. Os resultados da classificação a partir da inspeção visual podem ser resumidos na tabela 2.

Tabela 2. Sintetização da distribuição de isoladores nas classes estabelecidas.

\begin{tabular}{|c|c|}
\hline Classes & Número de Isoladores \\
\hline A & 14 \\
\hline B & 21 \\
\hline C & 25 \\
\hline Total & 60 \\
\hline
\end{tabular}

Os resultados oriundos dos ensaios em laboratório são apresentados e discutidos a seguir.

Ensaios em laboratório e extração de atributos

A partir dos ensaios em laboratório, mais de 2 horas de vídeo oriundas da inspeção por detecção de radiação ultravioleta foram registradas; cerca de 180 imagens térmicas foram geradas e 90 minutos de áudio foram armazenados. O processo de extração de atributos foi realizado computacionalmente.

\section{Classificação baseada em agrupamento difuso}

Os centroides relativos à inspeção por termografia infravermelha são apresentados na tabela 3. 
Tabela 3. Centroides dos clusters 1,2 e 3 para atributos oriundos das inspeções por termografia infravermelha.

\begin{tabular}{|c|c|c|c|}
\hline Atributos & Cluster 1 & Cluster 2 & Cluster 3 \\
\hline MédiaAum $\left({ }^{\circ} \mathrm{C}\right)$ & 2,0963 & 1,1628 & 1,8302 \\
\hline MédiaVar $\left({ }^{\circ} \mathrm{C}\right)$ & $1,6033 \times 10^{-4}$ & $-8,0468 \times 10^{-4}$ & $-0,0033$ \\
\hline MédiaVarVar $\left({ }^{(} \mathrm{C}\right)$ & $-0,0027$ & $-0,0021$ & $-0,0033$ \\
\hline MedianaAum $\left({ }^{\circ} \mathrm{C}\right)$ & 2,0637 & 1,1294 & 1,6730 \\
\hline MedianaVar $\left({ }^{\circ} \mathrm{C}\right)$ & $-0,0011$ & 0,0022 & $3,1468 \times 10^{-4}$ \\
\hline MedianaVarVar $\left({ }^{\circ} \mathrm{C}\right)$ & $-0,0012$ & $-0,0012$ & 0,0040 \\
\hline ModaAum $\left({ }^{\circ} \mathrm{C}\right)$ & 1,6910 & 0,8420 & 1,3566 \\
\hline ModaVar $\left({ }^{\circ} \mathrm{C}\right)$ & $-0,4483$ & $-0,4242$ & $-0,8002$ \\
\hline ModaVarVar $\left({ }^{\circ} \mathrm{C}\right)$ & $-0,7649$ & $-0,5486$ & $-0,9156$ \\
\hline VariânciaVarVar $\left({ }^{\circ} \mathrm{C}\right)$ & 0,0448 & 0,0378 & 0,0756 \\
\hline MaxLocalAum & 51,8476 & 44,0379 & 8,7726 \\
\hline MaxLocalVar & 53,3594 & 37,3370 & 5,6462 \\
\hline MaxLocalVarVar & 66,3588 & 40,3592 & 15,8529 \\
\hline MinAum $\left({ }^{\circ} \mathrm{C}\right)$ & 1,6731 & 0,7798 & 1,3233 \\
\hline CurtoseVarVar & 4,4438 & 3,6597 & 6,7935 \\
\hline AssimetriaVar & 0,1334 & $-0,1821$ & $-0,5619$ \\
\hline AssimetriaVarVar & $-0,2061$ & $-0,1562$ & $-0,3518$ \\
\hline AreaAum (uc. $\left.{ }^{\circ} \mathrm{C}\right)$ & 343,9013 & 190,7572 & 300,3376 \\
\hline AreaVar (uc..$\left.{ }^{\circ} \mathrm{C}\right)$ & 0,1340 & $-0,0207$ & $-0,4011$ \\
\hline AreaVarVar $\left(\right.$ uc. $\left.{ }^{\circ} \mathrm{C}\right)$ & $-0,0758$ & $-0,0958$ & $-0,1880$ \\
\hline
\end{tabular}

Uc $=$ unidade de comprimento (o comprimento dos isoladores foi parametrizado para 1).

Nota-se que o Cluster 1 apresenta maior aumento de temperatura médio e área abaixo da curva. Além disso, a elevação de temperatura mínima é a maior dentre os três clusters, bem como a moda. O Cluster 3 também apresenta valores elevados nesses atributos, com o Cluster 2 apresentando os menores valores. Assim, o Cluster 1 foi associado à classe $\mathrm{C}$, o Cluster 3 à classe B e o Cluster 2 foi associado ao estado operacional de menor grau de danos, classe A.

Para fins de exemplificação, o resultado do agrupamento é apresentado na figura 4. Cada elemento foi associado ao cluster que apresentou maior coeficiente de partição para facilitar a visualização. Os centroides de cada cluster foram indicados por $\times$.

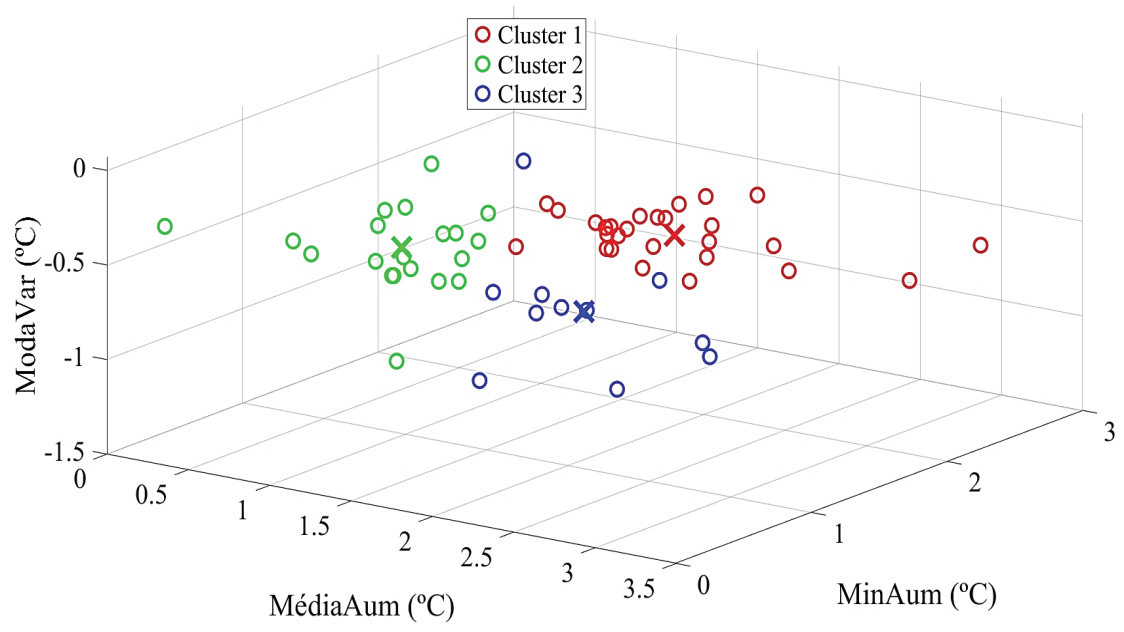

Figura 4. Ilustração dos clusters observados para dados oriundos das inspeções por termografia infravermelha. 
Os centroides obtidos para os atributos de radiação ultravioleta, por sua vez, podem ser observados na tabela 4.

Tabela 4. Centroides dos clusters 1, 2 e 3 para atributos oriundos das inspeções por detecção de radiação ultravioleta.

\begin{tabular}{|c|c|c|c|}
\hline Atributo & Cluster 1 & Cluster 2 & Cluster 3 \\
\hline Área ( $\mathrm{n}^{\circ}$ de pixels) & $1,4883 \times 10^{4}$ & $2,1522 \times 10^{3}$ & $1,6608 \times 10^{3}$ \\
\hline Perímetro ( $\mathrm{n}^{\circ}$ de pixels) & $4,4155 \times 10^{3}$ & $1,0329 \times 10^{3}$ & $1,0374 \times 10^{3}$ \\
\hline Persistência (1) & 5,5192 & 6,4402 & 22,8974 \\
\hline Fator de Forma (1) & 3,2952 & 1,9633 & 1,5678 \\
\hline
\end{tabular}

A partir da observação da tabela 4, nota-se que o Cluster 1 apresenta maior área, perímetro e, consequentemente, maior fator de forma. O Cluster 3, por sua vez, é o segundo em área e perímetro, destacando-se especialmente pela persistência elevada. O elevado valor da persistência pode ser associado a uma frequência mais constante de descargas, que pode estar relacionada ao início do processo de degradação do isolador. Desse modo, os Clusters 1,2 e 3 foram associados às classes $C, A$ e $B$, respectivamente.

A seguir, os resultados de agrupamento para dados de ruído ultrassônico são apresentados.

Tabela 5. Centroides dos clusters 1, 2 e 3 para atributos oriundos das inspeções por termografia infravermelha.

\begin{tabular}{|c|c|c|c|}
\hline Atributos & Cluster 1 & Cluster 2 & Cluster 3 \\
\hline $1^{\text {a }}$ Harmônica & $1,3074 \times 10^{-4}$ & $6,9954 \times 10^{-5}$ & $6,3946 \times 10^{-5}$ \\
\hline $2^{\text {a }}$ Harmônica & $9,0388 \times 10^{-6}$ & $2,4664 \times 10^{-6}$ & $2,8707 \times 10^{-6}$ \\
\hline $3^{\text {a }}$ Harmônica & $3,9529 \times 10^{-5}$ & $1,6317 \times 10^{-5}$ & $1,7502 \times 10^{-5}$ \\
\hline Média & $-8,5693 \times 10^{-7}$ & $-1,0781 \times 10^{-6}$ & $-9,8779 \times 10^{-7}$ \\
\hline$N^{\circ}$ de Zeros & $3,1490 \times 10^{5}$ & $3,0379 \times 10^{5}$ & $3,1046 \times 10^{5}$ \\
\hline $\begin{array}{c}\text { Coeficientes de } \\
\text { Variação }\end{array}$ & $-5,4482 \times 10^{3}$ & $-3,9225 \times 10^{3}$ & $-4,4040 \times 10^{3}$ \\
\hline Área abaixo da curva & 0,0289 & 0,0086 & $-0,0589$ \\
\hline Frequência Central & $2,5033 \times 10^{3}$ & $2,5032 \times 10^{3}$ & $2,5032 \times 10^{3}$ \\
\hline MédiaA1 & $-1,0711 \times 10^{-4}$ & $5,7033 \times 10^{-5}$ & $-4,8304 \times 10^{-4}$ \\
\hline MédiaA2 & $-1,5033 \times 10^{-4}$ & $9,7986 \times 10^{-5}$ & $-6,7199 \times 10^{-4}$ \\
\hline MédiaA3 & $-2,0960 \times 10^{-4}$ & $1,0646 \times 10^{-4}$ & $-9,1326 \times 10^{-4}$ \\
\hline AssimetriaA1 & 0,0463 & $-6,4789 \times 10^{-5}$ & $-0,0193$ \\
\hline AssimetriaA3 & $-0,0030$ & 0,0152 & $-0,0160$ \\
\hline MédiaD1 & $2,2797 \times 10^{-6}$ & $4,0386 \times 10^{-7}$ & $-8,7584 \times 10^{-7}$ \\
\hline MédiaD2 & $-1,5119 \times 10^{-6}$ & $1,9528 \times 10^{-5}$ & $-2,4568 \times 10^{-6}$ \\
\hline MédiaD3 & $-1,1947 \times 10^{-5}$ & $-5,1494 \times 10^{-6}$ & $-4,0243 \times 10^{-6}$ \\
\hline CurtoseD1 & 3,3041 & 3,3287 & 3,1676 \\
\hline AssimetriaD1 & $-0,0281$ & 0,0158 & 0,0065 \\
\hline AssimetriaD2 & 0,0157 & $-0,0095$ & 0,0163 \\
\hline AssimetriaD3 & 0,0580 & 0,0134 & $-0,0104$ \\
\hline
\end{tabular}

O Cluster 1 é o que apresenta maior média e maior valor da terceira harmônica (180 Hz), o que pode ser associado a descargas elétricas ocorrendo nos picos positivo e negativo da onda. Também é possível notar que, dentre os valores de assimetria para o primeiro nível da 
transformada Wavelet dos coeficientes de aproximação, o valor do Cluster 1 é o único positivo, o que indica uma cauda maior no lado direito da distribuição de probabilidade que, por sua vez, indica a presença uma frequência elevada de emissões acústicas de grande amplitude. A sua área calculada também foi a mais elevada, de forma que ele pode ser facilmente associado a um estado de degradação mais intenso (classe C).

Em grande parte dos atributos, não há uma divisão muito precisa entre os Clusters 2 e 3. É possível que os dados de $\mathrm{RU}$ utilizados forneçam informações limitadas para discernimento de estados de degradação iniciais, algo constatado também por Rocha (2014). Entretanto, ao observar pontos como a área e a assimetria negativa para o primeiro nível da transformada Wavelet dos coeficientes de aproximação, pode-se atribuir o Cluster 3 ao menor nível de degradação (classe A), de modo que o Cluster 2 é associado por eliminação a um nível intermediário (classe B).

Após o processo de extração dos outliers, agrupamento difuso e a comparação com a inspeção visual, os isoladores foram distribuídos nas classes propostas, de acordo com o apresentado na tabela 6.

Tabela 6. Apresentação de classes alocadas a partir de inspeção visual, agrupamento difuso e a rotulagem final.

\begin{tabular}{|c|c|c|c|}
\hline Classes & $\begin{array}{c}\text { Inspeção } \\
\text { Visual }\end{array}$ & $\begin{array}{c}\text { Agrupamento } \\
\text { difuso }\end{array}$ & $\begin{array}{c}\text { Rotulagem } \\
\text { final }\end{array}$ \\
\hline A & 13 & 36 & 11 \\
\hline B & 17 & 7 & 14 \\
\hline C & 21 & 8 & 26 \\
\hline
\end{tabular}

A partir da análise da tabela 6 , é possível observar que a aplicação do agrupamento difuso identificou a maior parte dos isoladores como pertencente à classe $\mathrm{A}$, de modo que se nota uma diferença considerável entre os dados obtidos a partir dos métodos de inspeção por detecção de radiação ultravioleta, termografia infravermelha e detecção de $R U$ e a inspeção visual. No entanto, a metodologia conseguiu identificar a necessidade de atribuir um grau mais severo de degradação a 5 isoladores, que outrossim não seriam retirados. Constata-se assim que a inspeção visual deve ser utilizada em conjunto com esses métodos.

\section{Conclusões}

Uma metodologia para redução da subjetividade na classificação de isoladores poliméricos foi desenvolvida. A metodologia tem como base o aprendizado de máquina não supervisionado e a inspeção visual, podendo ser utilizada de forma conjunta aos métodos convencionais, proporcionando maior confiabilidade na gestão técnica de isoladores em linhas de transmissão. Ademais, a classificação realizada, com menor grau de subjetividade, pode ter impactos significativos na aplicação de técnicas inteligentes baseadas em aprendizado supervisionado (mais precisamente na etapa de treinamento), o que representa um avanço no contexto de soluções inteligentes na área de alta tensão.

A importância da utilização da inspeção visual em conjunto com outros métodos foi constatada. Os defeitos identificados visualmente não foram evidenciados de forma satisfatória a partir dos agrupamentos de dados realizados, de modo que a inspeção visual ainda se apresenta como ferramenta essencial no diagnóstico de isoladores. 
Trabalhos futuros podem atestar os resultados deste trabalho a partir da submissão dos isoladores utilizados a ensaios para determinação da redução das suas características eletromecânicas, algo que não foi o foco do presente trabalho. Ademais, relações entre os métodos de inspeção e os defeitos nos isoladores podem ser investigadas de forma mais profunda.

\section{Agradecimentos}

Os autores agradecem à CAPES e ao CNPq pelo financiamento das bolsas de pesquisa e aos membros do Laboratório de Alta Tensão da Universidade Federal de Campina Grande - UFCG pelo suporte técnico-científico.

\section{Referências}

[1] R. MARTINS. Desenvolvimento de isolador polimérico inteligente. Curitiba: Departamento de Engenharia e Ciência dos Materiais. Tese de Doutorado. Universidade Federal do Paraná - UFPR, 2015.

[2] Y. G. PUSHPA, N. VASUDEV. Artificial Pollution Testing of Polymeric Insulators by CIGRE Round Robin Method - Withstand \& Flashover Characteristics. In: $3^{\text {rd }}$ International Conference on Condition Assessment Techniques in Electrical Systems (CATCON), 2017.

[3] A. KHALED, A. EL-HAG, K. ASSALEH. Equivalent Salt Deposit Density Prediction of Outdoor Polymer Insulators during Salt Fog Test. 2016 IEEE Conference on Electrical Insulation and Dielectric Phenomena (CEIDP), p. 786-789, 2016.

[4] Y. KIM, K. SHONG. The characteristics of UV strength according to corona discharge from polymer insulators using a UV sensor and optic lens. IEEE Transactions on Power Delivery, v. 26, n. 3, p. 1579-1584, 2011.

[5] I. M. A. M. KARUNARATHNA, K. T. L. KARUNATHILAKA, W. M. H. M. WANASINGHE, G. K. L. SAMPATH, J. R. LUCAS, R. SAMARASINGHE. Monitoring System for Outdoor Insulators. In: $20182^{\text {nd }}$ International Conference On Electrical Engineering (EECon), Colombo, 2018.

[6] K-E, B. BRITO. Extração de Atributos de Fáculas de Imagens Ultravioletas de Isoladores Poliméricos. [s.I.]. Dissertação (Mestrado em Engenharia Elétrica).Universidade Federal de Campina Grande, 2017.

[7] L. FANGCHENG, W. SHENGHUI, L. HEMING. Insulator pollution grade evaluation based on ultraviolet imaging and fuzzy logic inferenceAsia-Pacific Power and Energy Engineering Conference, APPEEC. Anais...2010

[8] S. K. POLISETTY, A. EL-HAG, S. JAYARAM. Classification of Common Discharges in Outdoor Insulation Using Acoustic Signals and Artificial Neural Network. High Voltage, p. 2-13, 2019.

[9] E. S. DOMINGUES. Improved Condition Monitoring of Composite Insulators. [s.I.] The University of Manchester, 2012.

[10] D. PERNEBAYEVA, A. IRMANOVA, D. SADYKOVA, M. BAGHERI, A. JAMES. High voltage outdoor insulator surface condition evaluation using aerial insulator images. High Voltage, v. 4, n. 3, p. 178-185, 2019.

[11] D. SONI. Supervised vs. Unsupervised Learning. Towards Data Science, 2018. Disponivel em: https://towardsdatascience.com. Acesso em: 17 de Junho de 2020.

[12] C. P. G. GUEDES. Classificação das Condições de Degradação de Isoladores Poliméricos pelos Níveis de Radiação UV e a Lógica Fuzzy. [s.I.] Universidade Federal de Campina Grande, 2015.

[13] G. R. LIMA. Aprimoramento da Classificação de Isoladores Poliméricos por Medições Termográficas e Radiação UV usando Processamento de Imagens e RNA. [s.I.] Universidade Federal de Campina Grande, 2017.

[14] T. V. FERREIRA, A. D. GERMANO, E. G. COSTA. Ultrasound and artificial intelligence applied to the pollution estimation in insulations. IEEE Transactions on Power Delivery, v. 27, n. 2, p. 583-589, 2012.

[15] P. H. V. ROCHA. Estimação da poluição de isoladores em linhas de transmissão a partir da irradiação de RF. Tese (Doutorado em Engenharia Elétrica). Universidade Federal de Campina Grande - UFCG. Campina Grande, 2017.

[16] P. H. V. ROCHA, E. G. COStA, A. R. SERRES, G. V. R. XAVIER, J. E. B. PEIXOTO, R. I. LINS. Inspection in overhead insulators through the analysis of the irradiated RF spectrum. Electrical Power and Energy Systems, v. 113, 2019. 
[17] B. DIAS. Gestão Técnica de Isoladores Poliméricos Utilizando Aprendizado de Máquina. [s.1]. Tese de Doutorado. Universidade Federal de Campina Grande - UFCG, 2021.

[18] W. PEDRYCZ. Knowledge-Based Clustering. Haboken, Nova Jersey: John Wiley \& Sons, Inc., 2005.

[19] R. XU, D. C. WUNSCH II. Clustering. Haboken, Nova Jersey: John Wiley \& Sons, Inc., 2009.

[20] R. S. GORUR. Condition Assessment of Polymer Insulators. PSERC Seminar Presentation, 2006, p. 37.

[21] T. V. FERREIRA, A. D. GERMANO, E. G. COSTA. Ultrasound and artificial intelligence applied to the pollution estimation in insulations. IEEE Transactions on Power Delivery, v. 27, n. 2, p. 583-589, 2012.

[22] FlorentinO, M. T. B.; COSTA, E. G. DA; FERREIRA, T. V.; GERMANO, A. D. Spectral subband centroid energy vectors algorithm and artificial neural networks for acoustic emission pattern classification. Advances in Electrical and Computer Engineering, v. 19, n. 3, p. 49-56, 2019.

[23] E. HANCER, B. XUE, M. ZHANG. A survey on feature selection approaches for clustering. In: Artificial Intelligence Review, v. 53, 2020.

[24] M. HAINDL, P. OMOL, D. VERVERIDIS, C. KOTROPOULOS. Feature selection based on mutual correlation. In: Carrasco Ochoa JA, Kittler J, Martínez-Trinidad JF (eds) Progress in pattern recognition, imageanalysis and applications. Springer, Berlin, pp 569-577, 2006. 